\title{
STABILOMETRIC ASSESMENT OF POSTURAL CHANGES INDUCED BY SPINE ORTHOSES IN A GERIATRIC POPULATION
}

Orthopaedic

Molfetta L

University Of Genoa - School Of Medical And Pharmacological Sciences - DIMI Department Of Internal Medicine And Medical Specialities -research Center Of Osteoporosis And Osteoarticular Disease-Genoa - Italy

Seriolo C*

University Of Genoa - School Of Medical And Pharmacological Sciences - DIMI Department Of Internal Medicine And Medical Specialities -research Center Of Osteoporosis And Osteoarticular Disease-Genoa - Italy * Corresponding Author

Casabella A

University Of Genoa - School Of Medical And Pharmacological Sciences - DIMI Department Of Internal Medicine And Medical Specialities -research Center Of Osteoporosis And Osteoarticular Disease - Genoa - Italy

Massaro W

University Of Genoa - School Of Medical And Pharmacological Sciences - DIMI Department Of Internal Medicine And Medical Specialities -research Center Of Osteoporosis And Osteoarticular Disease - Genoa - Italy

Palermo A

IRCCS Istituto Auxologico Italiano - 3 Unit Of Orthopaedic Surgery, Capitanio Hospital, Milan, Italy

\section{Leandri}

University Of Genoa - School Of Medical And Pharmacological Sciences - DINOGMI Department-Genoa - Italy

\section{ABSTRACT}

The use of spine orthoses in vertebral fractures of frailty in the adult-elderly population always poses problems of appropriate choice of the same as a function of the control of patients' balance. The vertebral fracture conjugated to the use of external devices modifying the posture can lead to negative effects, starting from the patient's poor compliance. The study of the equilibrium with dynamic platform represents a moment of validation of the appropriateness of the corset, even if temporarily carried. This has implications also for the rehabilitation of these patients after surgery.

\section{KEYWORDS}

Fracture, Spine, Osteoporosis, Orthosis, Stabilometry

\section{INTRODUCTION}

Fractures of the spine, the main complication of osteoporosis in the female gender, make dorsal hypercifosis with pain and rigidity. The progressive decrease in vertebral body height changes the structure and postural balance of the subject and can induce other falls with a severe fracture progression (1). The spinal orthoses in fractures and secondary hypercifosis are a therapeutic antalgic and mechanical compensation support (2). To the postural alteration of the fractured column are added the stresses induced by the mechanical orthesis which tries to stabilize a rigid and painful rachis. The postural reaction to the corset is variable and correlates with the general conditions, with the functional status of the patient and with the fracture or its outcomes (3)

In this study we verified the influence of corsets in vertebral postural balance, through the response to a complex, multidirectional, destabilizing dynamic stimulus, induced by the dynamic stabilometric platform, to identify the reactivity of the subject and therefore the influence of the corsets themselves, towards the patient and in comparison with each other (4).

\section{MATERIALS AND METHODS}

16 female patients, aged between 60 and 80 (72 \pm 0.5 years), were enrolled for the study who has been carried out an anamnestic evaluation (Tab.1). Autonomous women were admitted to the study, without the use of supports or other orthoses, without trauma or aftereffects, without neurological illness, without alteration of vision and hearing.

The subjects then performed a dynamic stabilometric examination using the Dynamic Stabilometric Balance Platform (GeaHD), which registers the vertical forces, in particular the weight force; it allows to check the postural adaptability of the subject through a multifactorial evaluation of motor, proprioceptive and vestibular integration. The platform is equipped with a compressed air system that allows the processing of evaluation parameters of the tracks including the XY coordinates of the pressure center (COP) in the horizontal plane; the length of the oscillations (distance by the COP); the average speed (VM) of the movements; the surface of the ellipse (dispersion of the oscillations), the spectrogram (frequency of oscillations (sagittal and frontal), the state kinesigram (ground representation of the oscillations), and the stabilogram (graph of the movement in time of the COP). TLMs having the following mechanical characteristics:

Tilting Hyperextensor Corset(TLM35) in aluminum, characterized by three thrusts, anterior sternal, anterior iliac and posterior dorsal, the latter corrective of the dorsal hypercifosis;

-Spinfast, semi-rigid thoraco-lombo-sacral anterior-posterior traction corset with shoulder straps, with removable and manually moldable spinal plate;

- Modular DorsoKit Corset with low lumbar component (DorsoKit A) and with additional stabilization device on the back with antimicrobial tie rods (DorsoKit B).

As for the analysis of the dynamic part, we divided the stabilomentric path into 3 different times that correspond to different phases of the stimulus: the first time ( $\mathrm{t} 0-0.5 \mathrm{~s}$ ) that goes from 0 to 0.5 seconds corresponds to the phase pre-stimulus; the second time ( $\mathrm{t} 0.5-2 \mathrm{~s}$ ) ranging from 0.5 to 2 seconds, corresponds to the stimulus phase and finally the third time ( $\mathrm{t} 2-5 \mathrm{~s})$ that goes from 2 to 5 seconds corresponds to the post-stimulus phase. The execution of static and dynamic stabilometric tests on the platform took place in 5 different ways: standard evaluation in an upright position without a corset with arms dangling and looking forward; evaluation with Tilting Hyperextensor Corset for vertebral collapses (TLM-35); evaluation with Thoracolumbar Corset with shoulder straps and spinal plate (SpinFast); evaluation with Lombo-Sacral Dynamic Corset (DorsoKit A); evaluation with Thoraco-Lombo-Sacral Corset with shoulder straps (DorsoKit B). 
The assessments of the subject were divided into 4 times by 6 registrations each.

In the first recording the subject opened his eyes and the platform was in a static position ( $\mathrm{OA} /$ valve all off).

In the second recording the subject closed his eyes and the platform was always in a static position (OC/valve all off).

In the third recording the subject opened his eyes and the platform was in dynamic position (OA/ valve $\mathrm{v} 1 \mathrm{v} 4 \mathrm{on})$.

In the fourth recording the subject closed his eyes and the platform remained in dynamic position ( $\mathrm{OC} / \mathrm{valve} \mathrm{v} 1 \mathrm{v} 4 \mathrm{on}$ ).

Data from GeaHD were transferred to a software to filter and process them. The software used for experimentation was designed and developed in the LabView (C) proprietary language of the National Instruments. The signals acquired are the following: 4 signals of the respective load cells, for determining the projection of the body barycentre on the support surface (COP); 1 synchronism signal with the stimulus; 2 signals for monitoring the inclination of the platform by means of special gyroscopic transducers; 4 electromyographic signals. Finally, a statistical study of the data was carried out with direct export to a printout readable in Excel for the evaluation of the significance of the results and related correlations. For the purposes of statistical analysis, the mean quadratic root (rms) of the stabilometric trace in the time domain is the most effective and most used parameter in the stabilomentric analyzes since it is a reliable measure of the oscillation variability of the subject. However, during the drafting of the work, we preferred to use as an effective parameter the maximum oscillation (Max Sway) in the antero-posterior and lateral-lateral direction (Max Y Sway and Max X Sway), since these are situations in which destabilizing stimuli of impulsive type, the platform has reported more effective values.

\section{RESULTS}

All the subjects participated in the test responding to the induced stimulus.

Tab. 2 and 3 show the data of the evaluations with the platform in a static position (valve all off). It is clear that the oscillations of the subjects on the lateral-lateral plane in the first half of the stabilometric tracing (Max X Sway I) with open eyes, were greater with the DorsoKit A and Dorso Kit B corsets than the Corset C35. As summarized in Tab. 3 , in subjects in the condition of a fixed platform with eyes closed, it should be noted that the oscillations on the anterior-posterior plane are greater with the use of the corset $\mathrm{C}-35$ with respect to DorsoKit A, and to DorsoKit B. DorsoKit A corset is the best choice of stability under closed eye conditions and non-moving stability platform. Tables 4 and 5 show the results with moving platform (valve $\mathrm{v} 1 \mathrm{v} 4 \mathrm{on}$ ). It is noted that the major displacements occur during recordings without corsets. About the corsets used, the statistically greater oscillations on the lateral-lateral plane, concerned the recordings in which the subject wore the $\mathrm{C} 35$, followed successively by the DorsoKit B in favor of the Spinfast corset (Tab 4). Finally, with regard to the recordings made with the eyes closed with the platform in operation, we note that even in this case the major oscillations on the lateral-lateral plane occurred when the subjects were not equipped with corsets.

\section{DISCUSSION}

The corset is a device applied to the outside of the spine and exerts a mechanical action, with multiplanar stresses on the individual sagittal curves, aimed at achieving a therapeutic purpose, ie the support of the rachis in the consolidation phase of the fracture or in the management of results post-traumatics. Applied a corset, the paravertebral muscular system adapts itself to the corrective actions of the corset itself with the spine in a rigid, forced posture and correction above all of the sagittal curves. However, the application of the corset by itself exerts an action at the same time disturbing the mechanical arrangement of the trunk; the stresses that it induces, related to its design and its mechanical conception, can favor imbalances with an effect opposite to the compensation objective for which it is used. The corsets generate a moment of extension of the spine, increasing the perception of body posture through biofeedback, improving it. This results in a strengthening of the musculature of the spine, a more stable balance and a reduction of pain, with an increase in functional capacity and with the improvement of the parameters of quality of life (5)
In the presence of a thoracic vertebral fracture or thoraco-lumbar passage, the corset supports the pharmacological therapy, decreasing the axial stresses on the fracture through a corrective action on the sagittal curves with a reduction of axial stresses on the fracture. The result is a decrease in pain and a decrease in the wedge deformation of the vertebra. The use of the corset in vertebral fractures or in posttraumatic results changes to some extent the balance of the subject in orthostasis and in walking especially. A six-month treatment with spinal orthosis showed no significant differences in back pain, back force force, or kyphosis index among the analyzed groups. In the spinal orthosis group, vertebral pain decreased slightly and the force of the extensors increased by $26.9 \%$, demonstrating a dynamic role as coach (6). A multicenter, prospective, randomized comparison study between a thoracolombosacral orthosis (TLSO) and no orthoses (NO) in the treatment of acute $\mathrm{A} 3$ thoracolumbar fractures has shown that the two treatments that follow a similar management protocol are equivalent to 3 post-trauma months. After 5- 10 years (mean follow-up $7.9 \pm 1.1$ years), patients treated without corset with early mobilization maintain the same pain relief (7). By studying the reaction to a sudden multiplanar dynamic stimulus, in orthostasis on the stabilometric platform it was possible to document which of the selected corsets had an action of greater control of the oscillations and therefore generally of control of the vertebral column (8).

The study found that patients without corsets all had destabilizing oscillations, reflecting a valid base reactivity. The lumbar corset Dorsokit A has proven to be the one with the lowest mechanical interaction about stiffening and restraining the spine, unable to stop the oscillations.

The $\mathrm{C} 35$ has resulted the device characterized by the greater rigidity and ability to contain the spine; the sternal thrust balanced by the vertebral pelota constitutes an element of very strong mechanical interaction, as shown by the fact that the oscillations on the anteriorposterior plane were greater than the Dorsokit B and Dorsokit A. Also on the lateral-lateral plane the oscillations, statistically more significant concerned the $\mathrm{C} 35$, followed successively by the DorsoKit $\mathrm{B}$ in favor of the Spinfast corset (Tab 4). Greater oscillation expresses less control of the spine, armed with the specific guardian; in any case, they are high guardians with a thoracic and vertebral socket. Significant and noteworthy was the data related to the evaluation with closed eyes, qualifying moment of the study of equilibrium; the major fluctuations on the lateral-lateral plane occurred when the subjects were not equipped with corsets, with a free trunk, where brain control did not count on any peripheral stimulation of pre-alarm or control by the guardians.

This data finds comfort in literature. A meta-analysis of 4 randomized controlled trials revealed no significant differences between the Spinomed orthosis, the stiff corset and the soft corset in acute fractures. Therefore, it may be useful to recommend medium-term use of the spinal-type orthosis to patients with subacute fracture. (9)

Among the various systematic reviews capable of providing meaningful information on the use of corsets, we cite the study by Karimi (10). The author analyzed nine studies including two randomized controlled parallel group studies, four randomized crossover studies, two before-and-after studies and a parallel group observational study. The analysis of the results evaluated the impairments, the motor activities and the patient's participation, with contrasting results and of limited quality. These were 21 retrospective studies with variable follow-up. However, it emerged that in subjects with a thoracolumbar fracture they had a good functional recovery and work activity. The use of the orthosis had not influenced the angulation of kyphosis in subjects with stable fracture in the thoracolumbar spine. The positive effects of orthoses would be mainly on immobilization, on protection and on stabilization of the spine.

Newman et al. in a systematic review including 12 studies, 8 RCTs or pilot RCTs and 4 non-randomized studies out of 626 participants showed that the semi-rigid thoracolumbar orthosis could help control pain, strength, posture and quality of life, with a particular influence on the balance for an orthosis of weighted kyphosis (11).

In conclusion, the use of spine orthoses in general and in vertebral fractures of fragility in particular is not supported by statistically consolidated work in relation to the specific role, especially to the control of fracture deformity. Numerous studies are necessary for this purpose. 
Tab.1:Principal patients characters

\begin{tabular}{|l|l|l|l|l|}
\hline $\mathrm{N}^{\circ}$ & Mean Age (years) & Weight $(\mathrm{Kg})$ & Height $(\mathrm{m})$ & $\mathrm{N}^{\circ}$ shoes $(2 / 3 \mathrm{~cm})$ \\
\hline
\end{tabular}

\begin{tabular}{|r|l|l|l|l|}
\hline 1 & 61 & 62 & 1,68 & 36 \\
\hline 2 & 73 & 51 & 1,52 & 37 \\
\hline 3 & 73 & 56 & 1,50 & 35 \\
\hline 4 & 71 & 60 & 1,52 & 36 \\
\hline 5 & 62 & 55 & 1,50 & 35 \\
\hline 6 & 77 & 60 & 1,60 & 39 \\
\hline 7 & 79 & 69 & 1,70 & 38 \\
\hline 8 & 76 & 82 & 1,56 & 37 \\
\hline 9 & 73 & 55 & 1,50 & 36 \\
\hline 10 & 66 & 63 & 1,60 & 37 \\
\hline 11 & 62 & 78 & 1,62 & 38 \\
\hline 12 & 78 & 51 & 1,55 & 35 \\
\hline 13 & 69 & 55 & 1,53 & 37 \\
\hline 14 & 74 & 70 & 1,70 & 41 \\
\hline 15 & 74 & 62 & 1,56 & 36 \\
\hline 16 & 80 & 64 & 1,51 & 39 \\
\hline
\end{tabular}

Tab.2: risultati della valutazione con valve all off / $O A$

\begin{tabular}{|l|c|c|}
\hline OA - VALVE ALL OFF & Max X Sway I & Mean Y Tot \\
\hline test t c35 VS dorso kitA & 0,0413 & 0,0000 \\
\hline Mean evaluation c35 & 1,6112 & $-5,3381$ \\
\hline Mean evaluation dorso kitA & 1,9248 & $-0,3434$ \\
\hline test t c35 vs dorso kit B & 0,0343 & 0,0137 \\
\hline Mean evaluation c35 & 1,6112 & $-5,3381$ \\
\hline Mean evaluation dorso kit B & 1,9093 & $-2,1237$ \\
\hline
\end{tabular}

Tab.3: Evaluation result on valve all off / OC

\begin{tabular}{|l|c|c|c|c|}
\hline OC-VALVE ALL OFF & Y rms I & Y rms II & Yrms III & Y rms tot \\
\hline test t c35 VS dorso kit A & 0,0106 & 0,0148 & 0,0070 & 0,0071 \\
\hline Mean evaluation c35 & 9,2682 & 9,5589 & 9,7002 & 9,7085 \\
\hline Mean evaluation dorso kit A & 6,4354 & 7,1235 & 6,9676 & 7,0571 \\
\hline test t c35 vs dorsokit B & 0,0157 & 0,0131 & 0,0061 & 0,0067 \\
\hline Mean evaluation c35 & 6,7627 & 7,2164 & 7,0309 & 7,1758 \\
\hline Mean evaluation dorso kit B & 9,2682 & 9,5589 & 9,7002 & 9,7085 \\
\hline
\end{tabular}

Tab.4: Evaluation result on valve v1v4 on / OA

\begin{tabular}{|l|c|c|}
\hline OA - VALVE V1V4 ON & Max Y Sway III & Max X Sway III \\
\hline test t standard VS c35 & 0,0128 & 0,0413 \\
\hline Mean evaluation standard & 21,0250 & 12,8955 \\
\hline Mean evaluation c35 & 17,4875 & 10,3276 \\
\hline test t standard VS spinfast & 0,0065 & \\
\hline Mean evaluation standard & 21,0250 & \\
\hline Mean evaluation spinfast & 17,0550 & \\
\hline test t standard VS dorso kit B & 0,0027 & \\
\hline Mean evaluation standard & 21,0250 & \\
\hline Mean evaluation dorso kit B & 16,8372 & \\
\hline
\end{tabular}

Tab. 5: Evaluation result on v1v4 on / OC

\begin{tabular}{|c|c|c|c|c|}
\hline $\begin{array}{c}\text { OC - VALVE V1V4 } \\
\text { ON }\end{array}$ & X rms I & X rms II & $\begin{array}{c}\text { X rms } \\
\text { III }\end{array}$ & $\begin{array}{c}\text { X rms } \\
\text { tot }\end{array}$ \\
\hline $\begin{array}{c}\text { test t standard VS } \\
\text { c35 }\end{array}$ & 0,0228 & 0,0077 & 0,0321 & 0,0102 \\
\hline $\begin{array}{c}\text { Mean evaluation } \\
\text { standard }\end{array}$ & 11,4779 & 13,7014 & 12,7528 & 13,2634 \\
\hline Mean evaluation c35 & 8,3408 & 10,0152 & 9,7649 & 9,8988 \\
\hline $\begin{array}{c}\text { test t standard VS } \\
\text { dorso kit B }\end{array}$ & 0,0049 & 0,0170 & & \\
\hline $\begin{array}{c}\text { Mean evaluation } \\
\text { standard }\end{array}$ & 10,1318 & 29,2301 & & \\
\hline $\begin{array}{c}\text { Mean evaluation } \\
\text { dorso kit B }\end{array}$ & 7,0216 & 23,4428 & & \\
\hline
\end{tabular}

\section{REFERENCES}

1) Seriolo B, Paolino S, Casabella A, et al. Osteoporosis in the elderly. Aging Clin Exp Res. 2013 Oct;25 Suppl 1:S27-9

2) Goodwin VA, Hall AJ, Rogers E, et al. Orthotics and taping in the management of vertebral fractures in people with osteoporosis: a systematic review. BMJ Open. 2016 May 4;6(5)

3) Della Volpe, R., Popa, T., Ginanneschi, F., et al. Changes in coordination of postural control during dynamic stance in chronic low back pain patients. Gait Posture. 2006;24(3):349-55
4) Nardone, A, Giordano, A, Corrà, T., et al. Responses of leg muscle in humans displaced while standing - Effect of types of perturbation and of postural set. Brain. 1990 Feb;113 (Pt 1):65-84

5) Pfeifer M, Gehlen M, Hinz C. Spinal orthoses in the treatment of vertebral fracture with osteoporosis : A systematic review article]. Z Rheumatol. 2017 Dec;76(10):860-

6) Kaijser Alin C, Uzunel E, Grahn Kronhed AC, et al. Effect of treatment on back pain and back extensor strength with a spinal orthosis in older women with osteoporosis: a randomized controlled trial. Arch Osteoporos. 2019 Jan 9;14(1):5 -8

7) Urquhart JC, Alrehaili OA, Fisher CG, et al. Treatment of thoracolumbar burs fractures : extended follow-up of a randomized clinical trial comparing orthosis versus no orthosis. J Neurosurg Spine 2017 Jul;27(1):42-47

8) Wu, G., Haugh, L., Sarnow, M. et al. A neural approach to motor-sensory relation during postural disturbance. Brain Research Bulletin Brain Res Bull. 2006 ;69(4):365-74.

9) Jin YZ, Lee JH. Effect of Brace to Osteoporotic Vertebral Fracture : a Meta-Analysis. J Korean Med Sci. 2016 Oct;31(10):1641-9

10) Karimi M. The effects of orthosis on thoracolumbar fracture healing: A review of the literature. JOrthop. 2015 Nov 17;12(Suppl2):S230-7

11) Newman M, Minns LC, Barker K. Spinal Orthoses for Vertebral Osteoporosis and Osteoporotic Vertebral Fracture : A Systematic Review. Arch Phys Med Rehabil. 2016 Jun;97(6):1013-25 